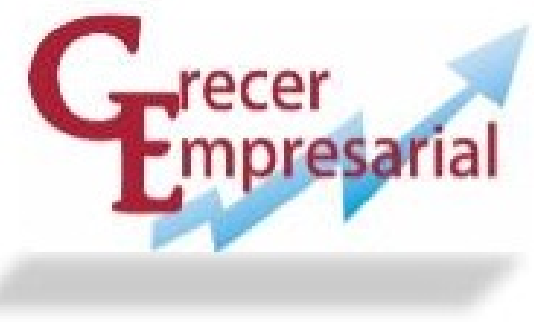

\title{
PRIORIZACIÓN DE PRODUCTOS AGROINDUSTRIALES PARA LA TRANSFORMACIÓN PRODUCTIVA: UN ANÁLISIS DESDE EL COMERCIO.
}

Cristian Samir Ulloa Ramos ${ }^{1}$, Vanesa Alejandra Molina Fresneda ${ }^{2}$, María Alejandra Puentes Talero ${ }^{3}$, Lilian Andrea Rayo Maldonado ${ }^{4}$

Artículo Enviado: 14/06/2017; Aceptado: 11/07/2018; Publicado: 02/12/2019

\section{Resumen}

El país se ha enfocado desde finales de la década pasada en la transformación productiva, que tiene como finalidad fortalecer los procesos de generación de valor y diversificar la oferta exportadora de las empresas en el mercado internacional. Este trabajo pretende identificar los productos de mayor potencial exportador en el sector agroindustrial colombiano, que serían base para la transformación productiva del país, en cuanto permitirían una consolidación en el proceso de internacionalización. Se concluye que, a pesar de mantener una balanza comercial negativa, tanto a nivel general como para el sector agroindustrial, el país cuenta con productos que a través del tiempo mantienen superávits comerciales como lo son: frutas y fruto de cáscara (excepto las nueces de aceite), fresco o seco, café y sucedáneo del café y materiales vegetales crudos. Esta priorización permitiría que las empresas y el gobierno centren sus recursos en la creación de valor agregado para dichos productos, diferenciándolos y posicionándoles en otros mercados.

Palabras claves: Transformación productiva; Balanza comercial; Sector agroindustrial; Competitividad; Industrialización.

\footnotetext{
1 Docente e Investigador Principal. Programa de Negocios Internacionales. Facultad de Ciencias Económicas y Administrativas. Universitaria Agustiniana - UNIAGUSTINIANA. Economista, Universidad Nacional de Colombia - Sede Bogotá. Magíster en Desarrollo, Instituciones e Integración Económica de la Universitat de Valencia, España.

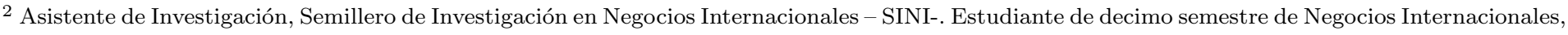
Universitaria Agustiniana - UNIAGUSTINIANA.

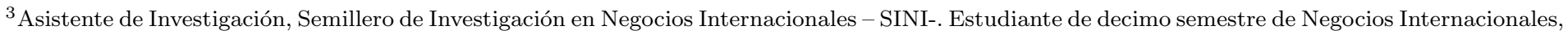
Universitaria Agustiniana - UNIAGUSTINIANA

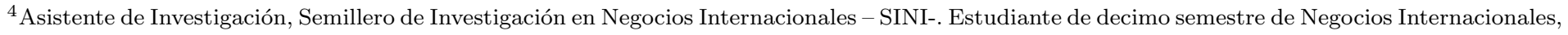
Universitaria Agustiniana - UNIAGUSTINIANA.
}

UlloA RAMOS, C. S., MOLINA FRESNEDA, V. A., RAYO MALDONADO, L. A., \& PUENTES TALERO, M. A. (2019)

Este es un artículo open Access bajo Licencia Creative Commons Atribución-No Comercial-Sin Derivadas 4.0 Internacional. 


\title{
PRIORITIZATION OF AGRIBUSINESS PRODUCTS FOR PRODUCTIVE TRANSFORMATION: AN ANALYSIS FROM TRADE.
}

\begin{abstract}
Since the end of the last decade, the country has focused on the productive transformation, which aims to strengthen the processes of generating value and diversify the export offer of companies in the international 1 market. This paper aims to identify the products with the greatest export potential in the Colombian agribusiness sector, which would be the basis for the productive transformation of the country, as they would allow a consolidation in the internationalization process. It is concluded that, despite maintaining a negative trade balance, both at a general level and for the agribusiness sector, the country has products that over time maintain commercial surpluses such as: fruits and nuts (except oil nuts), fresh or dried, coffee and coffee substitutes and raw plant materials. This prioritization would allow companies and the government to focus their resources on the creation of added value for these products, differentiating them and positioning them in other markets.
\end{abstract}

Keywords: Productive transformation; Balance of trade; Agribusiness sector;

Competitiveness; Industrialization.

\section{TRANSFORMATION PRODUCTIVE: UNE ANALYSE DU COMMERCE.}

\section{Résumé}

Le pays a mis l'accent depuis la fin de la dernière décennie dans la transformation de la production, qui vise à renforcer les processus de production et la valeur de diversifier les entreprises d'approvisionnement à l'exportation sur le marché international. Ce document vise à identifier les produits du plus grand potentiel d'exportation dans le secteur agro - industrie colombienne, qui serait la base de la transformation de la production du pays, permettrait une consolidation dans le processus d'internationalisation. Il est conclu que, malgré le maintien de la balance commerciale négative, globalement et pour le secteur de l'agro-industrie, le pays a des produits au fil du temps à maintenir des excédents commerciaux tels que les fruits et les noix (l'exclusion des noix huile), frais ou secs, succédanés de café et de café et matières végétales brutes. Cette hiérarchisation permettrait aux entreprises et au gouvernement de concentrer leurs ressources sur la création de valeur ajoutée pour ces produits, en les différenciant et en les positionnant sur d'autres marchés.

Mots-clés: Transformation productive; Balance commerciale; Secteur agro-industriel; Compétitivité Industrialisation

UlloA RAMOS, C. S., MOLINA FRESNEDA, V. A., RAYO MALDONADO, L. A., \& PUENTES TALERO, M. A. (2019)

Este es un artículo open Access bajo Licencia Creative Commons Atribución-No Comercial-Sin Derivadas 4.0 Internacional. 


\section{APRIORIZAÇÃO DOS PRODUTOS AGROINDUSTRIAIS PARA A TRANSFORMAÇÃO PRODUTIVA: UMA ANÁLISE DESDE O COMÉRCIO}

\section{Resumo}

O país concentrou-se desde finais da década passada na sua transformação produtiva, a qual tem por finalidade o fortalecimento dos processos geradores de valor e de diversificação da oferta exportadora das empresas no merado internacional. Este trabalho pretende identificar os produtos com o mais grande potencial exportador no setor agroindustiral colombiano, os quais seriam a base para a transformação produtiva do país, em tanto permitirão a consolidação do processo de internacionalização. Conclui-se que a pesar de manter a balança comercial negativa, tanto a nível geral, quanto no setor agroindustrial, o país conta com produtos que ao longo do tempo mantem superavits comerciais tais como: as frutas e frutos de cáscada (por exemplo nozes de óleo), fresco ou seco, café, substituto do cafe e materiais vegetáis crus. Esta prioriozação vai permitir que as empresas e o governo foquem seus recursos na criação de valor agregado para ditos produtos, os diferenciado-os e posicionando-os em outros mercados.

Palavras-chave: Transformação produtiva; Balança comercial; setor agroindustrial; Competitividade; Industrialização.

\section{Introducción}

La definición de ventajas comparativas de las empresas en comercio internacional, se describen a partir de los costes relativos y su dotación de factores productivos. Colombia, es un país que se caracteriza por tener una amplia oferta de recursos agropecuarios derivada de su ubicación geográfica. El clima ecuatorial, los diversos pisos térmicos y la dotación importante de recurso hídrico, son entre otras, razones para definir las ventajas que tiene el país en el mercado internacional.

El país durante las últimas tres décadas ha dado mayor importancia al desarrollo de sectores como el minero-energético, que tiene una importancia relativa mayor en el comercio internacional, pero que no ha permitido el crecimiento de otros sectores que podrían llegar a ser igualmente competitivos. Sin embargo, la estructura productiva del país y su crecimiento económico no han permitido su desarrollo productivo, en sectores como el agropecuario e industrial.

La agricultura e industria son sectores que vienen rezagados en el crecimiento de la producción nacional, resultados que no se compadecen con las ventajas comparativas que podrían explotarse para su desarrollo. Además, la inversión pública y privada en dichas actividades económicas no ha privilegiado los productos de mayor potencial exportador, sumado a una baja capacitación de agricultores y empresarios para la creación de valor y mayor conocimiento del mercado internacional.

Este trabajo pretende mostrar el balance comercial y la evolución de la canasta exportadora del país, con el fin de identificar los tres grupos de productos con mayor potencial en el comercio. Se concluye que el país debería priorizar la producción, creación de valor y transformación productiva en pro de productos agroindustriales como: frutas y frutos de cáscara fresca

UllOA RAMOS, C. S., MOLINA FRESNEDA, V. A., RAYO MALDONADO, L. A., \& PUENTES TALERO, M. A. (2019)

Este es un artículo open Access bajo Licencia Creative Commons Atribución-No Comercial-Sin Derivadas 4.0 Internacional. 
o seca (excepto las nueces de aceite), café y sucedánea del café y materiales vegetales crudos.

El documento se encuentra organizado en cuatro apartados. El primero describe el fundamento teórico referido a la teoría de ventaja comparativa, tanto en su descripción clásica como en la descripción práctica de revelar ventajas en el comercio. El segundo describe el tipo de investigación y las principales actividades desarrolladas para llegar a dichos resultados parciales. El tercero muestra principales resultados de la investigación, en principio en relación con las balanzas comerciales y, luego, con la descripción de la balanza para los tres grupos de productos con mayor potencial exportador. Por último, unas conclusiones y las referencias utilizadas.

\section{Marco Teórico}

\section{Modelo "Ricardiano" de ventaja comparativa}

Según Rosa (2012), el modelo de ventaja comparativa fue desarrollado por el economista David Ricardo (1772 - 1823), en donde plantea diferentes factores que determinan los patrones del comercio entre los países, como los son: costes unitarios relativos, la productividad del trabajo, los salarios relativos, entre otros.

En el mismo sentido Krugman y Obstfeld (2011), mencionan que los países participan en el comercio internacional por dos razones básicas, las cuales contribuyen a que obtengan ganancias del comercio. En primer lugar, porque los países son diferentes y en segundo lugar para conseguir economías de escala en la producción.

El modelo "Ricardiano" de ventaja comparativa indica que, si un país produce solamente una serie limitada de bienes, puede producir cada uno de esos bienes a una escala mayor, y por lo tanto más eficientemente que si intentara producir todo. Esto quiere decir que "un país tiene ventaja comparativa en su producción si el coste de oportunidad en la producción de dicho bien en términos de otros bienes es inferior en este país de lo que es en otros países" (Krugman \& Obstfeld, 2011, p.29).

\section{Ventaja comparativa revelada}

De acuerdo con García, Martínez, Díaz y Molina (2012), la ventaja comparativa es un componente teórico para explicar los orígenes del comercio internacional. Esta se basa en las primeras teorías expuestas por Ricardo y Heckscher y Ohlin. La ventaja comparativa se mide a través de los índices de Ventaja Comparativa Revelada, los cuales se calculan a partir de la observación al comercio y sus patrones, permitiendo así, identificar dicha ventaja. Se resalta que los índices de ventaja comparativa revelada, miden únicamente la ventaja comparativa en productos exportados y no en aquellos que sin ser comercializados internacionalmente podrían desarrollar la ventaja, de ahí el calificativo de revelada.

Según el Instituto Interamericano de Cooperación para la Agricultura (2011), este indicador de ventaja comparativa revelada fue creado por el economista Liesner (1958), quien planteó la idea de utilizar el rendimiento relativo de las exportaciones como índice de ventaja comparativa. Sin embargo, Balassa, economista húngaro, en 1965 adoptó la idea de Liesner, denominándola como índice de ventaja comparativa revelada por primera vez. Este agregó el uso de la proporción de cuotas derivadas de las exportaciones, dando lugar a la segunda versión del índice de ventaja comparativa, siendo este uno de los índices de rendimiento más citado entre otros:

Horbath (2005),Faustino (2008), D i Maio \& Tamagni (2007), Widgren (2005) y Macías (2010), así como también uno de los más utilizados actualmente. Este índice define la ventaja comparativa de un país

UlloA RAMOS, C. S., MOLINA FRESNEDA, V. A., RAYO MALDONADO, L. A., \& PUENTES TALERO, M. A. (2019)

Este es un artículo open Access bajo Licencia Creative Commons Atribución-No Comercial-Sin Derivadas 4.0 Internacional. 
" $\mathrm{i}$ " en un bien " $\mathrm{j}$ " como:

$$
I V C R_{i j}=\left(\frac{X_{i j}}{X_{j}}\right) /\left(\frac{X_{t}}{X}\right)
$$

Donde $I V C R_{i j}$ y es el índice de ventaja comparativa revelada del país $i$ en el bien $j, X_{i j}$ representa las exportaciones del país $i$ del producto $j \mathrm{y} X_{j}$ se refiere a las exportaciones mundiales del producto $j \mathrm{y}$ en donde $X_{t}$ son todas las exportaciones totales del país $i$, mientras $X$ representa a las exportaciones totales del resto de los países" (García, Martínez, Díaz y Molina, 2012, p.80).

Para esta investigación se empleará la versión del índice RCA dado por Balassa el cual "mide el grado de importancia de un producto dentro de las exportaciones de un país a otro, versus la importancia de las exportaciones del mismo producto en las exportaciones del mismo país hacia el mundo" (Lima y Alvarez, 2012, p.24).

\section{Metodología}

El proyecto es una investigación en curso, apoyada por el Semillero de Investigación en Negocios Internacionales de la Universitaria Agustiniana UNIAGUSTINIANA. Se define como una investigación de tipo cuantitativo con un alcance descriptivo. Se caracteriza por ser secuencial y de carácter probatorio, ya que establece hipótesis de contraste con variables cuantitativas y describe su evolución en un periodo determinado.

Inicialmente, se realizó la búsqueda de los datos sobre las exportaciones de los productos a analizar, por ello se utilizó la base de datos de la "Conferencia de las Naciones Unidas sobre Comercio y Desarrollo" (UNCTAD).

Con estos datos se realizó el cálculo de participación por sector de la balanza comercial tanto a nivel general como para el sector y cada producto.
El cálculo de la balanza comercial se realiza para el periodo 1995-2015. De acuerdo a la ecuación establecida:

$$
\begin{aligned}
& B C=X-M \\
& B C=X_{n}-M_{n} \\
& B C=X_{j}-M_{j}
\end{aligned}
$$

Donde $\mathrm{BC}$ es la balanza comercial total, $\mathrm{X}$ se refere a las exportaciones totales del país hacia el mundo, y M a las importaciones totales. Para cada caso se calculó también las balanzas para sector y producto, el subíndice n significa la variable para el sector agroindustrial y el subíndice i referido a los productos específicos.

Se priorizaron tres productos a partir del volumen de exportación total entre 1995 y 2015. Los tres grupos de productos elegidos son aquellos que mostraron mayores valores en la sumatoria de exportaciones en el periodo de estudio, teniendo en cuenta que fuesen parte del sector agroindustrial colombiano.

\section{Resultados}

\section{Economía colombiana en la década de los} 90

La economía colombiana estaba caracterizada por tener un régimen de importaciones restrictivo, adicionalmente manejaba unos aranceles y sobretasas arancelarias que desincentivaban el ingreso de mercancías extranjeras. Existía un control administrativo "drástico" que aseguraba la protección de la economía del país, en el cual los bienes se clasificaban como importación prohibida, sujetos a licencia previa y aquellos que entraban de manera libre al Territorio Aduanero Nacional (TAN).

A inicios de esta década, la economía del mundo estaba cambiando, iniciando un proceso de "globalización económica", motivado por dos factores

UllOA RAMOS, C. S., MOLINA FRESNEDA, V. A., RAYO MALDONADO, L. A., \& PUENTES TALERO, M. A. (2019)

Este es un artículo open Access bajo Licencia Creative Commons Atribución-No Comercial-Sin Derivadas 4.0 Internacional. 
principales: 1. la entrada en una crisis económica y 2. La influencia de organismos internacionales, que promovían la integración entre países. Esto generó una apertura económica en la mayoría de los países tanto de la región como del resto del mundo.

Sin embargo, aunque Colombia no se vio afectada totalmente por los factores mencionados anteriormente, el país decidió iniciar su proceso de apertura económica, ya que esta era la dirección que estaba tomando el mundo en ese momento.

Desde el gobierno de Virgilio Barco Vargas (Presidente de Colombia 1986-1990), la apertura económica comenzó a ser un tema a tratar por los economistas de dicha administración, quienes asesoraron al entonces Ministro de Hacienda Cesar Gaviria Trujillo.

En el año 1990 a finales del mandato del presidente Barco, se expidió el CONPES 2465, titulado "Programa de Modernización de la Economía Colombiana", el cual impulsó la globalización del país. Sin embargo, la apertura económica en Colombia se visualizó aún más con el CONPES 2494, basado en la eliminación de la licencia previa y disminución de aranceles de forma gradual. No obstante, este proceso tuvo resultados negativos para el país, trayendo como consecuencia un aumento de la inflación y una disminución de las importaciones. Según Gaviria, esto sucedió debido a que el país no realizó el proceso correctamente, al desmontar gradualmente y no de manera inmediata los aranceles (Revista Dinero, 2013).

\section{Análisis de la balanza comercial de Colombia}

La economía colombiana se ha visto enmarcada por mantener una balanza comercial negativa a lo largo de su historia, debido a varios factores como: 1. Capacidad productiva insuficiente para abastecer la demanda nacional, 2. Fenómenos naturales desfavorables, 3. Menores costos de productos importados, 4. Variaciones constantes de la tasa de cambio, entre otros. Adicionalmente, las exportaciones de Colombia se caracterizan por proceder del sector primario, es decir, que su valor en el mercado no representa mayores ingresos para el país.

En la gráfica 1, se puede observar el comportamiento de las importaciones y exportaciones, y su respectiva balanza comercial durante los años 2006 - 2016; en la cual se puede evidenciar lo dicho anteriormente:

Grafica 1. Balanza comercial total 1995-2015

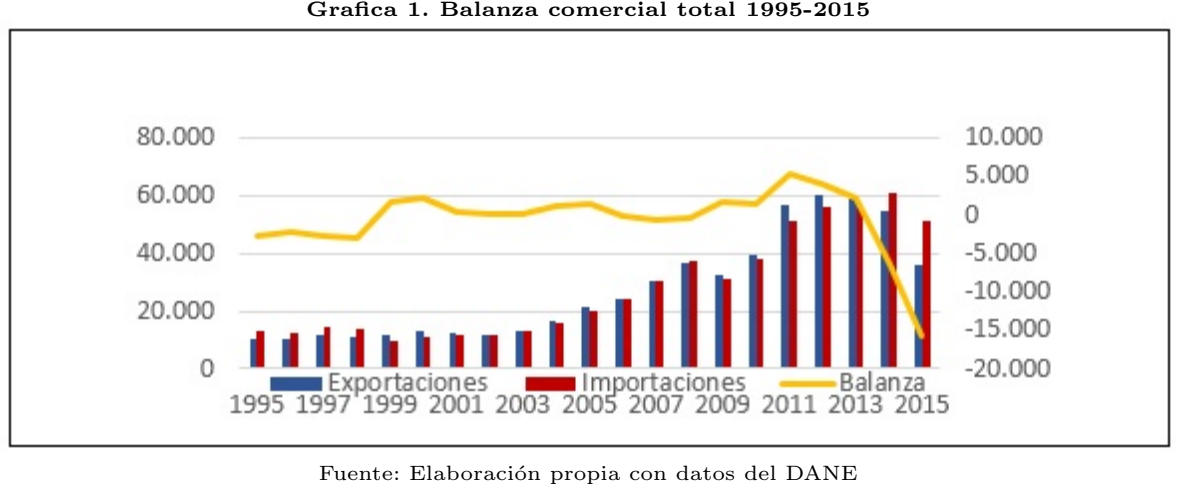

La apertura económica en el año 1991 generó un cambio en la estructura de la canasta exportadora del país, donde el sector Agro fue el más afectado; sin embargo, el presidente César Gaviria consideró que más que la apertura, lo que afectó a este sector fue una serie de acontecimientos adversos tales como: la sequía de 1992, el racionamiento, la caída de los precios internacionales y la decisión del Banco de la República

UllOA RAMOS, C. S., MOLINA FRESNEDA, V. A., RAYO MALDONADO, L. A., \& PUENTES TALERO, M. A. (2019)

Este es un artículo open Access bajo Licencia Creative Commons Atribución-No Comercial-Sin Derivadas 4.0 Internacional. 
(quien recientemente había adquirido su "independencia") de eliminar la entrega de subsidios a la producción agropecuaria. Teniendo en cuenta, que la economía del país estaba enfocada principalmente en la producción agroindustrial, se esperaba que esta tuviera un auge en las exportaciones gracias a la apertura económica.

No obstante, en las gráfica 2 se ve reflejada la disminución de participación de este sector en las exportaciones totales en el periodo del año 1995-2015, pasando del $40 \%$ en 1995 al $22 \%$ en 2015. Esto indica que evidentemente, este sector se ha visto afectado a lo largo del tiempo por diversas razones como: la influencia de la globalización en el desarrollo de otros sectores, principalmente el minero, el cual vio duplicada su participación en las exportaciones, teniendo en cuenta que la economía mundial se ha enfocado en la producción de bienes que requieren de materias primas derivadas de la extracción minera.

Por otra parte, la falta de inversión en el sector y el poco interés por la capacitación hacia los agricultores y transformadores, no ha permitido generar el valor agregado a los productos, por ende se limita el ingreso a nuevos mercados internacionales, siendo esto un reflejo del atraso en la industrialización del sector.

Gráfico 2. Participación por sectores en la canasta exportadora colombiana, 1995 y 2015

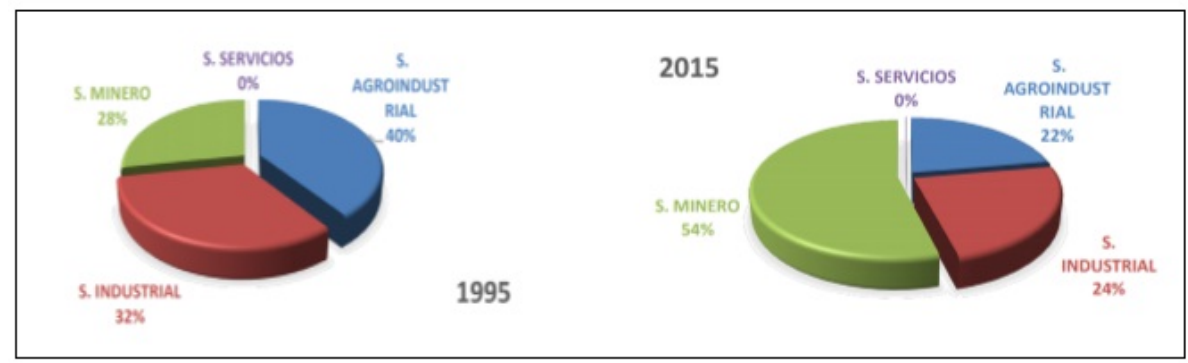

Fuente: Elaboración propia con datos de la UNCTAD

El sector minero fue el más beneficiado con este proceso, ya que, en las exportaciones totales del país, pasó de tener una participación en el año 1995 del $28 \%$, a una participación del $56 \%$ en el año 2015, obteniendo una variación positiva del $26 \%$ en 20 años. Lo anterior, se generó gracias a que la apertura económica fomentó el ingreso de la inversión extranjera directa mediante concesiones a multinacionales enfocadas a la extracción minera. Su objetivo fue estimular el desarrollo del sector minero, en un gobierno que ha promovido cambios normativos y ha abierto camino para la intensificación de la explotación minera, declarándola como una "actividad de utilidad pública y de interés social". Adicionalmente, este concedió títulos mineros en zonas protegidas como páramos, resguardos indígenas y territorios colectivos afrodescendientes. De los 114 millones de hectáreas que componen el territorio colombiano, más de 8,4 millones están concesionados para la exploración de minerales y más de 37 millones de hectáreas están titulados para la exploración de hidrocarburos. (Vicente, y otros, 2011)

Actualmente, el Registro Minero Nacional afirma que 1.717 empresas tienen títulos vigentes de concesiones para explorar o explotar minas, a las que se unen 7.200 títulos más, en manos de particulares. Hasta el 2002 las políticas estatales fueron relativamente conservadoras en términos de la concesión de títulos mineros, pero a partir de ese año comenzaron a incrementarse las solicitudes y concesiones. Entre 1990 y 2001 se entregaron en Colombia 1.889 títulos mineros (157 por año) y en 2010 ya había 8.928 concesiones (4.839.149 hectáreas) y 20.000 solicitudes en trámite. En potencias mineras latinoamericanas como Chile y Perú que hace años conviven con la minería a gran escala, el territorio concesionado asciende 13 millones y 27.1 millones de hectáreas respectivamente. Esto confirma por qué este

UlloA RAMOS, C. S., MOLINA FRESNEDA, V. A., RAYO MALDONADO, L. A., \& PUENTES TALERO, M. A. (2019)

Este es un artículo open Access bajo Licencia Creative Commons Atribución-No Comercial-Sin Derivadas 4.0 Internacional. 
sector ha venido presentando una variación positiva en la participación de las exportaciones totales del país, la cual se ilustra en las gráfica 2 (Vicente, y otros, 2011).

\section{Productos potenciales del Sector Agroindustrial}

A pesar de que este sector se ha visto relegado, Colombia aún continúa teniendo ventajas sobre algunos productos, donde ha logrado mantener una balanza comercial positiva, como se observa en la gráfica 3 , siendo el caso de los productos identificados por su respectiva nomenclatura arancelaria:

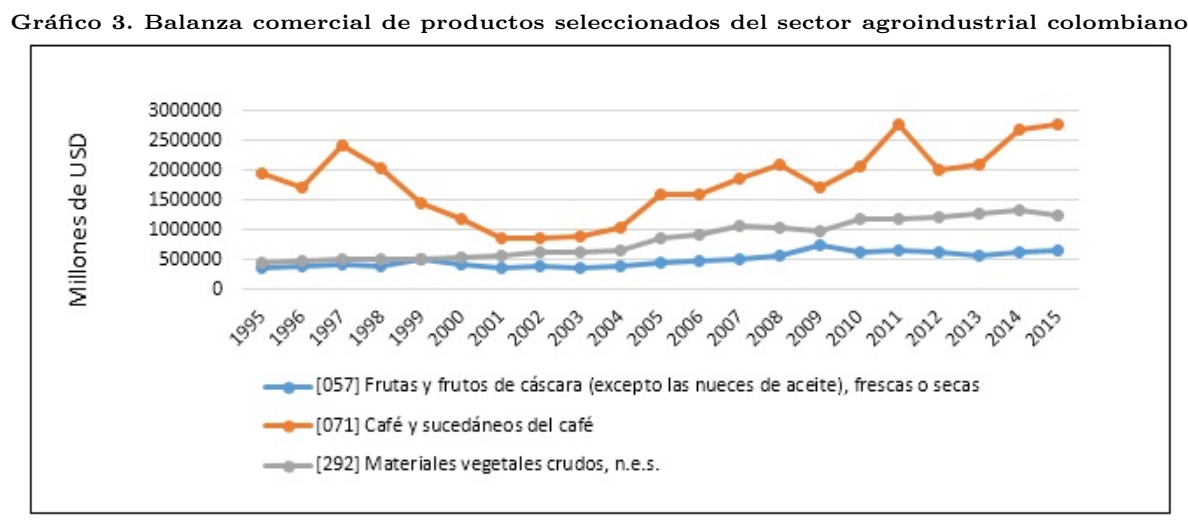

Fuente: Elaboración propia con datos de la UNCTAD

3.1. (057) Frutas y frutos de cáscara (excepto las nueces de aceite), frescas o secas

En base a los datos recolectados de la UNCTAD $^{5}$ , se logró identificar que la balanza comercial para este producto es positiva, lo cual se puede apreciar en la Gráfica 4. Esto se debe, a que Colombia se ha caracterizado por ser un país productor y exportador de frutas como: el banano, mandarina, naranja, aguacate, guayaba, piña, mango, mora, fresas, entre otros.

Colombia cuenta con una variedad de pisos térmicos que permiten el cultivo de diversos productos en diferentes regiones del país, siendo las más destacadas: el Urabá Antioqueño, Bolívar, Cesar, Córdoba, Cundinamarca, Huila, Magdalena y Tolima. (ElTiempo, 2008) Esto permite que Colombia tenga un alto potencial para competir en el mercado internacional. Sin embargo, este factor no se ha aprovechado debido a la falta de industrialización y tecnificación del sector, limitándose a la exportación del producto en su forma primaria, en consecuencia estos, no se pueden comercializar en el mercado internacional porque no cumplen los altos estándares de calidad que se exigen. Por la no transformación de dichos productos, se carece de un reconocimiento de marca.

Lo anterior, demuestra la baja capacidad productiva del país, que se debe a la falta de planeación para afrontar las adversidades climáticas que azotan durante el año al país; asimismo a la falta de infraestructura y una red vial interconectada que permita abastecer tanto el mercado local como los puntos logísticos de internacionalización de manera eficaz y a bajos costos.

\footnotetext{
${ }^{5}$ La Conferencia de las Naciones Unidas sobre Comercio y Desarrollo.
}

ULlOA RAMOS, C. S., MOLINA FRESNEDA, V. A., RAYO MALDONADO, L. A., \& PUENTES TALERO, M. A. (2019)

Este es un artículo open Access bajo Licencia Creative Commons Atribución-No Comercial-Sin Derivadas 4.0 Internacional. 


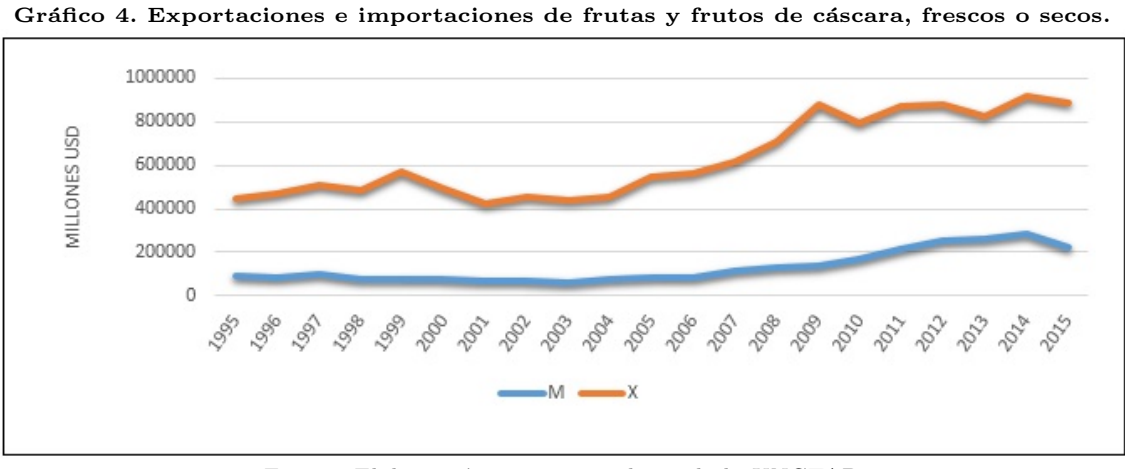

Fuente: Elaboración propia con datos de la UNCTAD

\section{2. (071) Café y sucedáneos del café}

El café ha sido el producto tradicional de exportación en Colombia a lo largo de su historia, por esta razón no es de extrañar que presente una balanza comercial positiva, la cual se puede apreciar en la Gráfica 5. Es importante resaltar que durante el periodo 2001 - 2004 se presentó una baja importante en su porcentaje de exportación, debido a la llegada de nuevos competidores al mercado.

La caída de la producción de café en Colombia ha sido un tema de importancia al interior del gremio cafetero. La pérdida de cerca del $30 \%$ en productividad en los últimos años, provocó que se estremeciera el análisis fundamental del mundo cafetero, obligando a muchos productores a adaptarse a un escenario de estrechez en la oferta. Lo anterior generó que, para mayo de 2012, la producción de café en Colombia alcanzara 689 mil sacos de 60 kilogramos, cifra que superó en un $2 \%$ el volumen producido el mismo mes del año anterior.

En los últimos años se ha incrementando la existencia de variables que están fuera del control de la autoridad cafetera, tales como el clima, que por varios años ha afectado severamente el territorio colombiano. Por esta razón, se viene capacitando a los cafeteros para que de forma ágil y eficaz sepan enfrentar de la mejor manera estas contingencias. Actualmente la caficultura colombiana está trabajando para ser climáticamente inteligente, es decir, que sus actividades sean capaces de adaptarse a la variabilidad climática.

La mentalidad de los cafeteros se encuentra enfocada en la renovación de cafetales resistentes al cambio climático, dando como resultado un proceso que a través de los años se ha propuesto metas a cumplir, alcanzando 72.849 hectáreas renovadas, es decir, 8,1\% del área total, en el año 2017. (Federación de cafeteros, 2018)

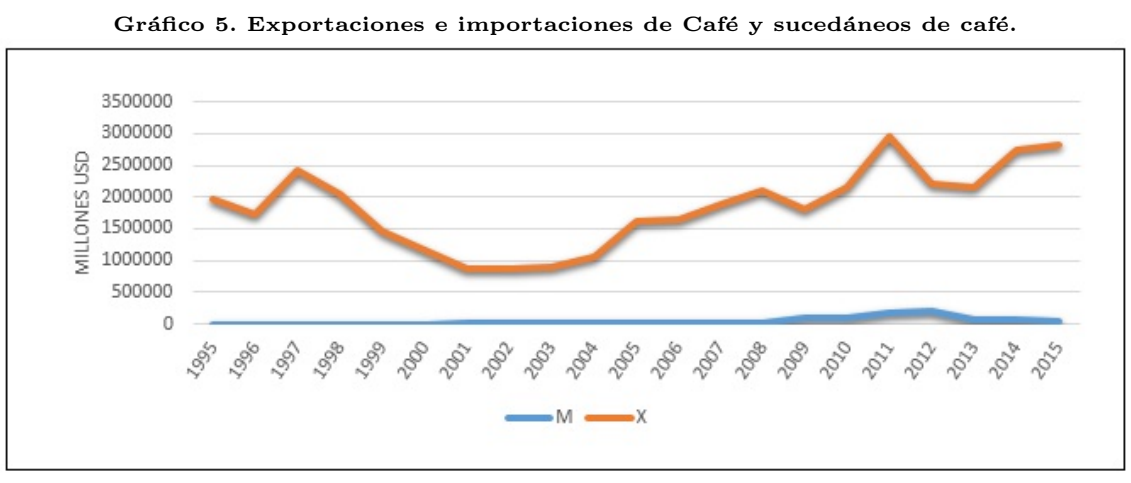

Fuente: Elaboración propia con datos de la UNCTAD

UlloA RAMOS, C. S., MOLINA FRESNEDA, V. A., RAYO MALDONADO, L. A., \& PUENTES TALERO, M. A. (2019)

Este es un artículo open Access bajo Licencia Creative Commons Atribución-No Comercial-Sin Derivadas 4.0 Internacional. 
El mercado del café es uno de los más importantes a nivel mundial, ya que no solo se consume en su forma primaria sino también en aquellos productos transformados, los cuales son de consumo masivo y utilizan como componente a este producto, tales como: bebidas energizantes, refrescos azucarados, medicamentos, postres, etc.

Por esta razón, es importante implementar un proyecto de transformación productiva, pues en el país no se ha aprovechado el potencial y la variedad de productos que se originan tan sólo de un grano de café. Asimismo, es importante resaltar que Colombia produce uno de los mejores cafés arábigos a nivel mundial con uno de los sabores más exquisitos, esto se convierte en una ventaja comparativa frente a sus principales competidores.

\section{3. (292) Materiales vegetales crudos}

Con base en los datos recolectados por la $\mathrm{UNCTAD}^{6}$, se logró identificar que la balanza comercial es positiva para este tipo de productos, lo cual se puede apreciar en la Gráfica 6. Esto se debe especialmente al cultivo de flores tipo exportación, las cuales se producen principalmente en tres regiones del país: Sabana de Bogotá, Antioquia y la zona del Eje cafetero.

Según el "Censo de fincas productoras de flores" realizado por el Departamento Administrativo Nacional de Estadística (DANE), la región de Bogotá cuenta con el clima ideal en el mundo para el cultivo de rosas y claveles. Por esta razón, la mayor concentración de cultivos de flores se encuentra en esta región hacia los municipios en el norte y los municipios de occidente, donde se cultivan rosas y claveles principalmente. En Rionegro, Antioquia, hacia el sector de Llano grande se encuentra la producción de crisantemos o pompones, así como de hortensias. Por último, en tierras cálidas y las regiones del Eje cafetero y Valle del Cáuca, se destaca la producción de flores tropicales o heliconias y el cultivo de anturios. (DANE, 2010)

En este grupo, también se destaca las exportaciones de plantas, partes de plantas, semillas y frutos de las especies utilizadas principalmente en perfumería, medicina o para usos insecticidas y parasiticidas.

Según la Revista Dinero (2017) en uno de sus artículos web, explica que históricamente el mercado de las flores colombianas ha abastecido el mercado de Estados Unidos, especialmente en celebraciones como el día de San Valentín, que es una de las fechas que más hace mover las exportaciones de ese producto a este país, el cual sigue ocupando el puesto número uno como destino de los tallos colombianos. Según el Centro Virtual de Negocios (CVN), Estados Unidos no es el único destino, también se destacan países como: Rusia, el Reino Unido, Japón, Holanda, España y Canadá. Las exportaciones de este producto, que ya es insignia del país, un incremento del $19 \%$ para el año 2010 lo cual representó un alza significativa.

En cuanto a la variedad de tallos ofrecidos por Colombia, las flores tropicales son un producto que tiene mucha demanda por sus características, pues son flores atractivas y cargadas de colores vivos para los clientes. Además de las rosas, los claveles y las flores tropicales, también se destacan otros productos ornamentales, como las alstroemerias, hortensias y crisantemos. (Revista Dinero, 2017)

Igualmente, en este grupo se encuentran muchos otros productos potenciales para optimizar su producción, logrando tener una mayor participación en el mercado internacional, gracias a sus características las cuales se relacionan directamente con su lugar de origen, es así como se destacan: bambúes, raíces de ginseng, bejucos, variedad de semillas, entre otros.

${ }^{6}$ Ibíd.

UlloA RAMOS, C. S., MOLINA FRESNEDA, V. A., RAYO MALDONADO, L. A., \& PUENTES TALERO, M. A. (2019)

Este es un artículo open Access bajo Licencia Creative Commons Atribución-No Comercial-Sin Derivadas 4.0 Internacional. 
Gráfico 6. Exportaciones e importaciones de materiales vegetales crudos, n.e.s.

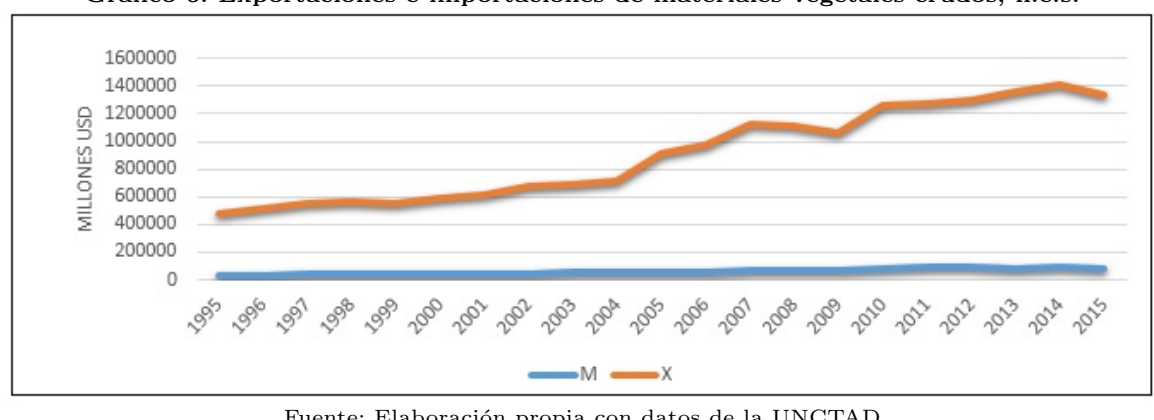

Todos los productos mencionados en el anterior análisis, son potenciales para realizar una transformación productiva, la cual se podría llevar a cabo con la participación de entidades gubernamentales que promuevan y financien proyectos que los productores y transformadores tengan un apoyo solido a la hora de emprender.

El plan de transformación se debe caracterizar por el desarrollo de productos innovadores con altos estándares de calidad, que cumplan los requisitos y normas internacionales; que a su vez permitan la transformación del sector, pasando de un ciclo de producción y exportación de materias primas a uno de producción, transformación y comercialización, a través de la implementación del valor agregado que aumente la competitividad en mercados internacionales. Con esto, se espera lograr un desarrollo en la industrialización del sector, posicionándose como referente competente y eficiente tanto en el mercado nacional como en el internacional.

\section{Conclusiones}

El Programa de Transformación Productiva (PTP) del gobierno nacional, tiene como objetivo el fortalecimiento y promoción de la productividad y competitividad de las empresas colombianas. De acuerdo al PTP, el $34 \%$ de las exportaciones no minero-energéticas son tenidas en cuenta dentro del programa, en las cuales se encuentran las de servicios, manufacturas y agroindustria. Este programa se creó por la necesidad del país para generar una mayor sofisticación de su economía a través de la diversificación de su industria y agricultura, conduciendo al desarrollo productivo del país y forjando tanto un entorno más competitivo como empresas fuertes y productivas.

Este PTP está enfocado en diferentes sectores productivos del país, sin embargo, de los productos derivados de esta investigación, los materiales vegetales crudos no están incluidos dentro de lo Programa de Transformación Productiva para el sector "agroindustria".

Por lo tanto, a partir de los resultados de esta investigación, se obtendrá información relevante con relación al sector agroindustrial, la cual será determinante para que el gobierno guie el enfoque que debe tener la inversión del Estado, la inversión extranjera directa y los programas de transformación productiva que se están desarrollando para los productos que no están incluidos dentro del PTP expuesto por el Gobierno. Todo esto, con el objetivo de potencializar las exportaciones de los productos agroindustriales identificados en el análisis anterior y así lograr un avance en términos industrialización, tecnificación y competitividad del sector.

De igual forma, los agricultores colombianos se pueden apoyar y beneficiar de la información obtenida y plasmada en este documento, dado que a partir de

ULlOA RAMOS, C. S., MOLINA FRESNEDA, V. A., RAYO MALDONADO, L. A., \& PUENTES TALERO, M. A. (2019)

Este es un artículo open Access bajo Licencia Creative Commons Atribución-No Comercial-Sin Derivadas 4.0 Internacional. 
allí pueden identificar aquellos productos que posean un potencial exportador, además de permitirle analizar si su producción cuenta con algún producto de estas características.

\section{BIBLIOGRAFÍA}

Instituto Interamericano de Cooperación para la Agricultura (2011). Índice de ventaja comparativa revelada. Bogotá, Colombia. Recuperado de:

http://repiica.iica.int/docs/B0550e/B0550e3.html

DANE. (Junio de 2010). Censo de fincas productoras de flores. Recuperado el 2018, de https://www.dane.gov.co/index.php/estadisti cas-por-tema/agropecuario/censo-de-fincas-productoras-de-flores

El Tiempo. (12 de Diciembre de 2008). El Tiempo. Obtenido de El Tiempo: http://www.eltiempo.com/archivo/documento/CMS-47 20463

Federación de cafeteros. (2018). 2018, año de grandes desafíos para el sector y el gremio cafetero colombiano. Al Grano, 1.

Krugman, P. R., \& Obstfeld, M. (2011). Economía Internacional, Teoría y Política. Madrid: Pearson Addison Wesley.

Lima, J. y Alvarez, M. (2012). Indicadores de comercio exterior y política comercial: mediciones de posición y dinamismo comercial.(Documento de proyecto). CEPAL. Recuperado de: http://repositorio.cepal.org/bitstream/handle/11362/

3690/S2008794es.pdf

Garcia, F., Francisco , M., Díaz, H., \& Molina, M. (Octubre de 2012). Revista Estudiantil de Economía. Obtenido de TEC de Monterrey. Del sistema Tecnológico de Monterrey: http://ree.economiatec.com/A4N2/222282.pdf

Henry Rosa Polanco. (2012). redalyc. Recuperado el 2017, de http://www.redalyc.org/pdf/870/870253850

07.pdf

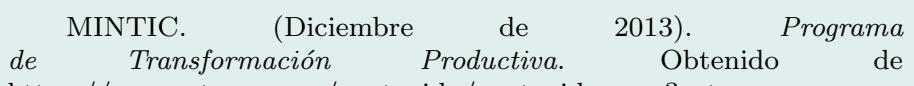
https://www.ptp.com.co/contenido/contenido.aspx?cat $\mathrm{ID}=607 \& \operatorname{conID}=1$

Revista Dinero. (2013). Revista Dinero. Obtenido de La apertura económica: http://www.dinero.com/edicion-impresa/caratula/arti culo/la-apertura-economica/182405

Revista Dinero. (2017). Flores colombianas retoman liderazgo en mercados extranjeros. Recuperado el 2017, de https://www.dinero.com/edicion-impresa/

negocios/articulo/sector-floricultor-de-colombia-en-mer

cados-extranjeros/245744UNCTAD. (s.f.).

UNCTADSTAT. Recuperado el Abril de 2017, de
http://unctadstat.unctad.org/wds/ReportFolders/

reportFolders.aspx?sCSChosenLang $=\mathrm{en}$

UNCTAD. (s.f.). UNCTADSTAT. Recuperado el Abril de 2017, de http://unctadstat.unctad.org/wds/

ReportFolders/reportFolders.aspx?sCSChosenLang=en

Valencia, A. (2012). Café de Colombia. Obtenido de Café de Colombia: http://www.cafedecolombia.com/cci-

fnc-es/index.php/comments/unamiradaalestadoactualya

lfuturodelaproducciondecafeencolombi/

Vicente, A., N. M., Slee, D. J., M. B., S. L., \& B. B. (Noviembre de 2011). Peace Brigades. Obtenido de Peace Brigades: http://www.peacebrigades.org/fileadmin/userfiles/ projects/colombia/files/colomPBIa/111122boletinfinal web.pdf

UlloA RAMOS, C. S., MOLINA FRESNEDA, V. A., RAYO MALDONADO, L. A., \& PUENTES TALERO, M. A. (2019)

Este es un artículo open Access bajo Licencia Creative Commons Atribución-No Comercial-Sin Derivadas 4.0 Internacional. 